\title{
Noninvasive Continuous Blood Glucose Monitoring by Dielectric Spectroscopy
}

\author{
Vignesh Balasubramanian, Scott Baskerville, Ryan Bailey, Keira McCarthy, Marc Rippen and John Burd*
} Alertgy, USA

Submission: May 13, 2021; Published: June 03, 2021

*Corresponding author: John Burd, Technical Advisor, Alertgy, 2412 Irwin Street, Melbourne Fl 32901, USA

\section{Abstract}

Aims: The Alertgy noninvasive continuous blood glucose monitor (NICBGM) is a novel wristband device that reports glucose levels without any entailing skin puncture. This study evaluated the performance of this device compared with an FDA-approved glucose meter in patients with type 2 diabetes.

Methods: The Alertgy DeepGluco NICBGM device measures changes in the dielectric spectrum it collects specific to blood glucose levels three times a minute. This spectral data is analyzed by using neural network analysis, machine learning and then with a calibration process is used to generate algorithms to estimate blood glucose (BG) values once the system is calibrated to the individual. The Roche Accuchek Inform II glucometer was used as a reference technique for calibration and then to determine the accuracy of blood glucose determinations. 27 patients completed three or more 120-minute sessions. Mean absolute relative difference (MARD) was calculated on the data collected.

Results: MARD values were compiled for two or more days of data collection following the first day of calibration. The MARD for all measurements was found to be 15.3 .

Conclusions: The resultant MARD suggest that this technique can achieve equivalent performance to that of existing CGM devices presently approved by the FDA when the same reference technique is utilized.

Keywords: Continuous glucose monitor; Glycemic control; Noninvasive glucose measurement; Diabetes

Abbreviations: NICBGM: Alertgy Noninvasive Continuous Blood Glucose Monitor; BG: Blood Glucose; DM: Diabetes Mellitus; FS: Fingerstick; T2DM: Type 2 Diabetes Mellitus, ESRD: End-Stage Renal Disease; POC: Point-of-Care; MARD: Mean Absolute Relative Difference; CGM: Continuous Glucose Monitor; GLP1: Glucagon-like Peptide-1; HbA1c: Hemoglobin A1c; ADHF: Acute Decompensated Heart Failure; SGLT2: Sodium Glucose Co-Transporter 2; DPP4: Dipeptidyl Peptidase-4; TZD: Thiazolidinedione

\section{Introduction}

Diabetes mellitus (DM) affects over 30 million people in the United States [1]. For most patients living with DM, frequent self-monitoring of blood glucose (BG) is needed for adequate outpatient glycemic control and has been associated with lower hemoglobin A1c [2]. The majority of patients with DM rely on fingerstick (FS) glucose measurements for self-monitoring, which necessitates lancing of the skin. FS testing is associated with pain and anxiety and can lead to nonadherence with home testing $[3,4]$. In recent years, the use of continuous glucose monitors (CGM) has become more widespread. CGMs significantly reduce the number of fingersticks needed for home monitoring; however, most devices still require the insertion of a new subcutaneous catheter every 10 to 14 days.

The Alertgy noninvasive continuous blood glucose monitor (NICBGM) is a novel device that does not entail skin penetration of the skin for continuous glucose monitoring. The device is worn on the wrist and measures BG levels through the use of dielectric spectroscopy. There have been several prior noninvasive BG monitors on the market; however, these have had limited success due to issues with inaccuracy and low reliability $[5,6]$. This study aimed to evaluate the performance of NICBGM retrospectively compared to an FDA-approved hospital-grade glucose meter in patients with T2DM.

\section{Materials and Methods}

\section{Study population}

This study was approved by the Complete Care Institutional Review Board. The study included 27 patients with T2DM recruited from the outpatient Complete Clinic. Inclusion criteria were known diagnosis of T2DM, current dietary or pharmacologic treatment for DM, recent hemoglobin A1c between $7.5 \%$ (58 $\mathrm{mmol} / \mathrm{mol}$ ) and $10.0 \%$ (86 mmol/mol), and age between 18 and 
75 years old. Exclusion criteria were prandial insulin use, fasting FS BG $<70$ or $>250 \mathrm{mg} / \mathrm{dL}$ on the day of study sessions, pregnancy (tested at the start of each study session), end-stage renal disease (ESRD), decompensated heart failure, medications that might cause false readings (including acetaminophen, ascorbic acid, dopamine, maltodextrin, or mannitol), and any conditions that might limit the NICBGM device, such as lesions on the forearms.

\section{Study protocol}

The studies were performed at the Complete Care main clinic location in Melbourne Florida. Two sets of trials were conducted to study the effect of using difference amounts and types of sugar. The Complete Care Trial I dataset had 17 unique subjects and 14 of those subjects suffered from type II diabetes among other comorbidities. All subjects had their baseline blood glucose measured every 5 minutes for 20 minutes before 68 grams of sucrose were ingested. After ingestion, blood glucose was measured every 5 minutes for 2 hours. All glucose measurements were carried out using the Accu-chek Inform II glucometer. Each 2 hour set of data was considered as an individual test. There were 60 individual tests that had followed this protocol and all 60 tests were included for further analysis.

The CompleteCare Trial II dataset had 10 unique patients, each of which being a diagnosed diabetic. CompleteCare Trial II subjects ingested 75 grams to 100 grams of dextrose for each of their respective tests. The total number of individual tests available were 45 . Patients participated in three or more visits, each with a 30 -min warm-up period and a study period lasting 120 minutes, at least three days apart, and most within 14 days from Day 1. At each visit, study subjects were fitted with the Alertgy NICBGM device for a warm-up period of 30 minutes before the start of data collection, and the device was worn throughout the duration of the 120-minute session thereafter (Figure 1). A trained technician took FS BG measurements. Baseline FSBGs were obtained at $-30 \mathrm{~min},-15 \mathrm{~min}$, and $0 \mathrm{~min}$ during the warm-up period. FSBG measurements were made before and during this warm-up period solely as health/safety checks to ensure that the patients were in adequate physiological condition to continue the study. These reference measurements were not paired with any NICBGM device estimates nor used within the machine learning training process.

Patients were instructed to fast for at least eight hours the night before. Patients were evaluated at the end of the study session to ensure that they were asymptomatic, and that their BG was in a safe range prior to discharge.

\section{Device technology}

FS BG was measured using the Accuchek Inform II glucose monitor to provide calibration values for the NICBGM. FS BG levels were entered into a secure database, and a proprietary calibration program was used to analyze the spectral data from the device during the training session on Day 1. These POC BG values were also used in calculations for the estimation of device accuracy. The NIBGM uses a weak electromagnetic field generated by its wristband sensor to produce dielectric spectra. The core sensing technology used is dielectric spectroscopy, which has been shown in prior work to be capable of noninvasively measuring blood glucose in a laboratory environment [7].

The monitoring technology implemented in the study includes a sensor having a multi-portion dielectric composite. A microstrip transmission line is placed on one surface of the dielectric composite and includes an input trace, radiator portion, and an output trace. The dielectric material adjacent to the radiator portion is selected for its dielectric properties such that it is matched with that of the target anatomy (distal forearm/wrist region) of the tested subject. This dielectric matching between the forearm and the sensor allows the radiator portion to effectively respond as if it were embedded inside the target anatomy, removing substantial uncertainty from the measurement process. By then applying a plurality of signals to the sensor, the signal's reflected and transmitted components can be measured and used to determine the amounts of certain constituents (including blood glucose) present in the subject's target anatomy. This study's dielectric sensor is capable of use in broadband sensing applications, ranging from $0 \mathrm{MHz}$ (DC) to $2 \mathrm{GHz}$. (United States Patent No. US20200217809A1, 2020).

The device sends and receives signals from the wrist area used to generate a dielectric spectrum once every twenty seconds. These spectral data are stored on the device and then downloaded wirelessly or through a USB port to a secure database. The spectral data are then inputted into a machine learning algorithm and used to create an estimate of the patient's BG level.

\section{Device algorithm}

Measurements for the algorithm training procedures were collected on each of the test subject's Day 1 trial. Blood glucose measurements were collected via the AccuCheck Inform II POC SMBG system with a 5-minute sampling period over a duration of 2 hours, resulting in 14 reference measurements per Day 1 session.

\section{Data transfer}

Alertgy received the complete FS BG dataset from Complete Care only for the first visit for each patient for calibration. For Days 2 and 3, Alertgy received only the -30 min FS BG data. The data were collected real-time. The data collected from the wristband were sent to the lab at Alertgy. Upon receipt in the lab, the data were processed from its original 'raw' form (consisting of many files of different types) and compiled into a format using internally-developed software modules. 


\section{Current Research in Diabetes \& Obesity Journal}

\section{Statistical methods}

Categorical patient characteristics were summarized using frequencies and percentages, while continuous measures were described with means and standard deviations, after assessing that they met normality assumptions. Mean absolute relative difference (MARD) was chosen as the measure of agreement between NICBGM findings and FS BG levels [7]. MARD was computed by taking the arithmetic mean of the absolute relative differences between the NICBGM system measures and the reference standard FS BG, which serves as the denominator of the calculation. The MARD is expressed as a percentage, and a lower MARD signifies better concordance between the two measurements

\section{Results}

NICBGM results are shown in the Following Figure 1.

Figure 1: Alertgy NICBGM results.

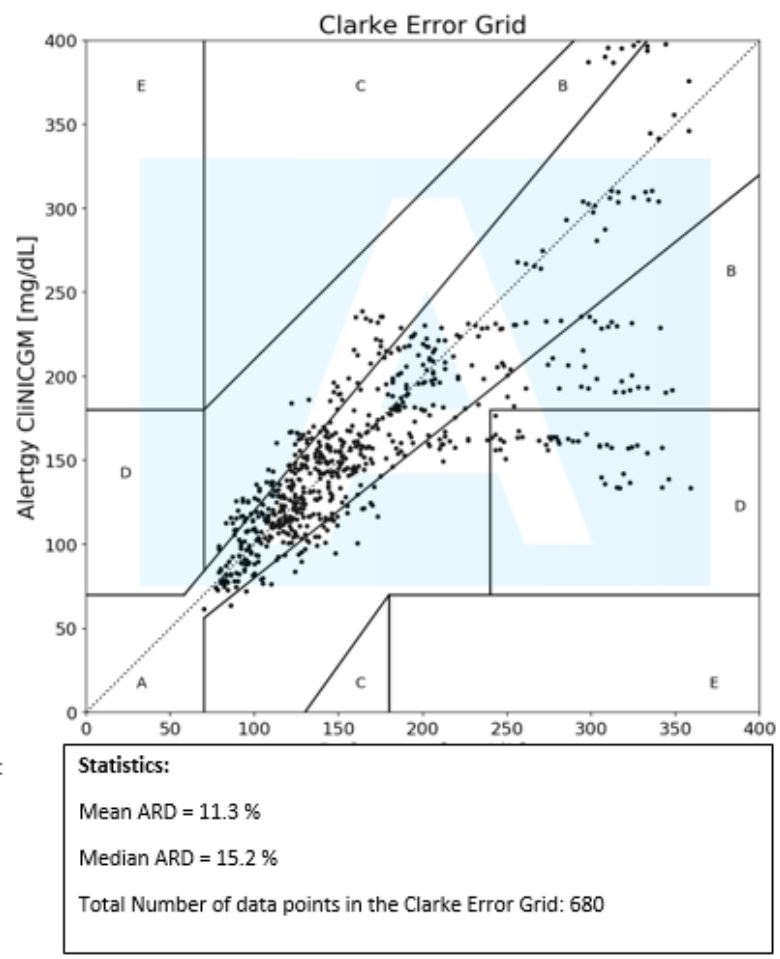

Table 1: Comparison of Alertgy NICBGM with CGMs.

\begin{tabular}{|c|c|c|c|c|}
\hline \multirow[t]{2}{*}{$\begin{array}{l}\text { DeepGluco }{ }^{\circledR} \mathrm{NICE} \\
\text { CompleteCare } \\
\text { Family Medicine }\end{array}$} & \multicolumn{4}{|c|}{$\begin{array}{l}\text { - Alertgy Data collected in Vivo } 27 \text { Patient Clinical Triaks by } 3^{*} \text { Party Medical Clinic } \\
\text { Analytical Range } 40-400 \mathrm{ug} / \mathrm{dl}\end{array}$} \\
\hline & $\begin{array}{l}\text { Dexcom } \\
\text { (c): }\end{array}$ & $\begin{array}{l}\text { Abbott } \\
\text { paten mol }\end{array}$ & $\begin{array}{l}\text { Senseonics } \\
\text { (tornesac) }\end{array}$ & Alertgy \\
\hline Invasiveness \& Sensor Placement & $\begin{array}{l}\text { Stin Plurces on } \\
\text { Nodomen }\end{array}$ & $\begin{array}{l}\text { Stin Fincese on } \\
\text { Upper Arm }\end{array}$ & 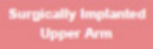 & 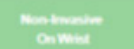 \\
\hline Measurand & $\begin{array}{l}\text { Geveose in hitesstad } \\
\text { Fhist }\end{array}$ & $\begin{array}{l}\text { Gucose in intestsad } \\
\text { Find }\end{array}$ & $\begin{array}{l}\text { Glucose in intertisid } \\
\text { Fund }\end{array}$ & Guncose in Hilood \\
\hline MARD (s) & $\omega$ & mor & $\mathrm{sr}$ & s \\
\hline Sensor Lifetime (days) & $\infty$ & u & so & steax \\
\hline Cost per Day in $\mathbf{s}$ us Dollars & - & 10 & $=10$ & $\Leftrightarrow 2$ \\
\hline
\end{tabular}




\section{Current Research in Diabetes \& Obesity Journal}

\section{Conclusion}

A comparative summary analysis of the results of the CompleteCare trial data generated by the Alertgy NICBGM are compared to other CGM devices in Table 1. Each listed device was evaluated against an SMBG system to develop the provided MARD values. The Alertgy NICBGM DeepGluco device provides equivalent accuracy. It also offers the advantages of no interstitial lag, non-invasive measurement, and a sensor life measured in years as opposed to days and is less costly to use.

\section{References}

1. National Diabetes Statistics Report (2020) Estimates of Diabetes and Its Burden in the United States. US Department of Health and Human Services. Centers for Disease Control and Prevention.

2. Miller KM, Beck RW, Bergenstal RM, Robin S Goland, Michael J Haller, et al. (2013) T1D Exchange Clinic Network. Evidence of a strong associa- tion between frequency of self-monitoring of blood glucose and hemoglobin A1c levels in T1D exchange clinic registry participants. Diabetes Care 36(7): 2009-2014

3. Olansky L, Kennedy L (2010) Finger-stick glucose monitoring: issues of accuracy and specificity. Diabetes care 33(4): 948-949.

4. Heinemann L (2008) Finger pricking and pain: a never ending story. Journal of diabetes science and technology 2(5): 919-921.

5. Vashist SK (2013) Continuous Glucose Monitoring Systems: A Review. Diagnostics (Basel) 3(4): 385-412.

6. Villena Gonzales W, Mobashsher AT, Abbosh A (2019) The Progress of Glucose Monitoring-A Review of Invasive to Minimally and Non-Invasive Techniques, Devices and Sensors. Sensors (Basel) 19(4): 800.

7. Karin Obermaier, Günther Schmelzeisen-Redeker, Michael Schoemaker, Hans-Martin Klötzer, Harald Kirchsteiger, et al. (2013) Performance evaluations of continuous glucose monitoring systems: precision absolute relative deviation is part of the assessment. J Diabetes Sci Technol 7(4): 824-832.

Your next submission with Juniper Publishers
will reach you the below assets
- Quality Editorial service
- Swift Peer Review
- Reprints availability
- E-prints Service
- Manuscript Podcast for convenient understanding
- Global attainment for your research
- Manuscript accessibility in different formats
( Pdf, E-pub, Full Text, Audio)
- Unceasing customer service
Track the below URL for one-step submission
https://juniperpublishers.com/online-submission.php

